\title{
Clusterizzazione di comorbidità e multimorbidità tra le persone che vivono con HIV, e rischio di interazione malattia-malattia: report preliminare. Clusterization of co-morbidities and multi-morbidities among persons living with HIV, and risk of disease- disease interactions: a preliminary report
}

\section{Carmen R. Santoro ${ }^{1}$, Elena Ricci ${ }^{2}$, Nicolò De Gennaro ${ }^{1}$, Chiara Bellacosa ${ }^{1}$, Marco Nofri ${ }^{3}$, Elisabetta Schiaroli ${ }^{3}$, Giancarlo Orofino ${ }^{4}$,Barbara Menzaghi ${ }^{5}$, Nicola Squillace ${ }^{6}$, Antonio Di Biagio ${ }^{7}$, Daniela Francisci ${ }^{8}$, Francesca Vichi ${ }^{9}$, Chiara Molteni $^{10}$}

${ }^{1}$ Clinica delle Malattie Infettive, Università degli Studi di Bari

${ }^{2}$ Fondazione IRCCS Ca' Granda Ospedale Maggiore Policlinico, Milano

${ }^{3}$ Dipartimento Medicina Interna e Medicina Specialistica 2, Clinica delle Malattie Infettive. Ospedale "Santa Maria della Misericordia". Azienda Ospedaliera di Perugia

${ }^{4}$ Malattie Infettive e Tropicali I, ASL Città di Torino

${ }^{5}$ UO Malattie Infettive, Ospedale di Busto Arsizio, ASST della Valle Olona

${ }^{6}$ Clinica Malattie Infettive, Ospedale Policlinico San Martino,Genova

${ }^{7}$ Dipartimento di Malattie Infettive ASST-MONZA, Ospedale San Gerardo, università di Milano-Bicocca, Monza

${ }^{8}$ Clinica delle Malattie Infettive. Azienda Ospedaliera "Santa Maria", Terni

${ }^{9}$ SOC malattie infettive, Ospedale Santa Maria Annunziata, Uslcentro, Firenze

${ }^{10}$ UOC di Malattie Infettive, Azienda Socio Sanitaria Territoriale di Lecco

\section{Riassunto}

La prevalenza di co-morbidità tra le persone che vivono con I'HIV è in crescita. Una questione importante riguarda l'associazione tra le co-morbidità (cioè la clusterizzazione), a causa delle implicazioni in termini di interazioni malattie-malattie e farmaco-farmaco. Lo scopo di questo studio è descrivere i pattern di co-morbidità e di multi-morbidità, la loro modalità di clusterizzazione e le potenziali interazioni malattia-malattia in una coorte di pazienti HIV.

Lo studio è un'analisi cross-sectional condotta dal Coordinamento Italiano per lo Studio di Allergia e Infezioni da HIV (CISAI) su pazienti adulti che afferiscono agli ambulatori HIV. Le mo-morbidità non-AIDS/non-HIV considerate includevano: malattie cardiovascolari, diabete mellito, ipertensione, malattia oncologica, broncopneumopatia cronica ostruttiva (BPCO), malattia epatica, disturbi psichiatrici e neurologici, malattia renale. La multi-morbidità è stata definita come presenza di due o più co-morbidità.

Sono stati arruolati 997 pazienti; 178 (17.8\%) non presentavano co-morbidità, mentre $275(27.6 \%)$ avevano una condizione e 544 (54.6\%) presentavano multi-morbidità: $25.8 \%$ avevano due condizioni, $19.6 \%$ tre, $6.6 \%$ quattro, $2.2 \%$ cinque, $0.4 \%$ sei. La co-morbidità più frequente era la dislipidemia (55.5\%), seguita da BPCO (30.0\%), infezione da

\section{Abstract}

The prevalence of co-morbidities, among persons living with HIV is increasing. An important issue is how the co-morbidities associate among them (i.e. clusterization of co-morbidities), because of the implications in terms of disease-disease and drug-drug interactions. Aim of the present study is to describe the patterns of co-morbidity and multi-morbidity, their clustering mode and the potential disease-disease interactions in a cohort of HIV patients.

The study is a cross-sectional analysis conducted by the Coordinamento Italiano per lo Studio di Allergia e Infezioni da HIV (CISAI) on adult subjects attending HIV-outpatient facilities. Non-AIDS/non-HIV co-morbidities considered included: cardiovascular disease, diabetes mellitus, hypertension, oncologic disease, osteoporosis, chronic obstructive pulmonary disease, liver disease, psychiatric illness, kidney disease. Multi-morbidity was defined as the presence of two or more co-morbidities.

997 patients have been enrolled. One hundred-seventy eight patients (17.8\%) had no co-morbidity, whereas 275 (27.6\%) had one condition and 544 (54.6\%) were multi-morbid: $25.8 \%$ had two co-morbidities, $19.6 \%$ three, $6.6 \%$ four, $2.2 \%$ five, and $0.4 \%$ six.
Autore

per la corrispondenza:

Carmen R. Santoro Clinica delle Malattie Infettive,

Università degli Studi di Bari Piazza Giulio Cesare, 11 70125 Bari.

carrisa@libero.it

Keywords:

HIV; co-morbidity; multi-morbidity;

disease-disease interactions; clusterization.

Potenziali conflitti di interesse:

nessuno.

JHA 2018; 3(4): 81-86

DOI: 10.19198/JHA31459 
HCV (25.6\%), ipertensione (21.9\%), malattia psichiatrica (10.2\%) e insufficienza renale (4.3\%).

Le diadi più frequentemente associate erano: 1$)$ dislipidemia + BPCO $(177,17.8 \%), 2)$ dislipidemia + ipertensione $(158,15.8 \%) ; 3)$ BPCO + HCV $(134,13.4 \%)$. Le triadi più frequenti erano invece: dislipidemia + BPCO + HCV (6.8\%), dislipidemia + ipertensione + BPCO (4.7\%) e dislipidemia + ipertensione + neoplasia (3.1\%). Pazienti con multi-morbidità erano più vecchi e mostravano parametri dell'infezione da HIV peggiori e dati metabolici più compromessi rispetto ai pazienti senza multi-morbidità.

I nostri dati mostrano che la presenza di co-morbidità è la regola nelle persone che vivono con l'HIV, e che la multi-morbidità si trova nella maggior parte dei pazienti che afferiscono ai centri del gruppo CISAl: erano presenti nei nostri pazienti diverse condizioni a rischio per interazioni maggiori (neoplasie + insufficienza renale, neoplasie + malattia epatica, malattia cardiovascolare + epatica) e minori (malattia cardiovascolare + insufficienza renale, malattia cardiovascolare + BPCO).

In conclusione, considerando l'invecchiamento delle persone che vivono con I'HIV, la loro persistente esposizione a infiammazione cronica e i possibili effetti della terapia antiretrovirale, è necessario affrontare fin d'ora il fenomeno della clusterizzazione, e identificare i pazienti a rischio di interazioni malattia-malattia.
The most frequent co-morbidity was dyslipidemia (55.5\%), followed by bronco-pulmonary chronic obstruction (COPD) (30.0\%), hepatitis C virus (HCV) infection (25.6\%), hypertension (21.9\%), psychiatric illness (10.2\%) and renal impairment (4.3\%).

Analyzing the pairs of co-morbidities, the most recurrent associations were: 1) dyslipidemia + COPD (177, 17.8\%), 2) dyslipidemia + hypertension (158, 15.8\%); 3) COPD + HCV infection (134, 13.4\%). The most recurrent triads were: dyslipidemia + COPD + HCV infection (6.8\%), dyslipidemia + hypertension + COPD (4.7\%) and dyslipidemia + hypertension + cancer (3.1\%). Multi-morbid patients were older and showed worse parameters of HIV infections, more compromised metabolic data with respect to non multi-morbids.

Our data show that co-morbidity is the rule among PLWH, and that multi-morbid patients are the majority. Moreover, about $30 \%$ of them had three or more chronic non-HIV related conditions. Several conditions at risk for mayor (cancer + renal impairment, cancer + liver disease, CVD + liver disease) and minor disease-disease interactions (CVD + renal impairment, CVD + COPD) were present among our patients. In conclusion, considering the aging of PLWH, their persistent exposure to chronic inflammation and the possible effects of antiretroviral therapy, it is mandatory to face since now the phenomenon of clusterization, and to identify all the patients at risk of disease-disease interactions.

\section{Introduction}

The prevalence of co-morbidities, among Persons Living With HIV (PLWH) infection is increasing in recent years as a consequence of aging, chronic inflammation, systemic immune activation, and long-term exposure to the combination antiretroviral therapy (CART) $(1,2)$. Consequently, increased attention has been paid to the treatment and the consequences of the major co-morbidities (cardiovascular, renal, neurocognitive, bone disease, cancer...). An important issue is how the co-morbidities associate among them (i.e. clusterization of co-morbidities), because of the implications in terms of disease-disease and drugdrug interactions. In fact, when the recommended therapy for treating one disease is contraindicated in the presence of another concurrent medical condition, this limits the usefulness of clinical practice guidelines, and makes managing the care of such patients challenging, especially in emergency conditions. In such scenarios, evidencebased treatment guidelines designed for single diseases can lead to serious therapeutic conflicts due to disease-disease interactions.
Aim of the present study is to describe the patterns of co-morbidity and multi-morbidity, their clustering mode and the potential disease-disease interactions in a cohort of PLWH.

\section{Patients and Methods}

The study is a cross-sectional analysis of baseline data from a cohort study, conducted by the Coordinamento Italiano per lo Studio di Allergia e Infezioni da HIV (CISAI). Patients analyzed were enrolled in the STOPS-HIV Study from April 2014 to October 2016: this is an ongoing cohort study including adult PLWH attending the HIV-outpatient facilities of the participating centers. In September 2018, in the frame if STOPS-HIV Study, the Cluster Project collected information from clinical records of these patients, in order to investigate comorbidities present at the time of enrollment.

Non-AIDS/non HIV co-morbidities considered included: cardiovascular disease (CVD) defined as previous diagnosis of myocardial infarction, stroke, angina pectoris, coronary artery, bypass grafting, and angioplasty and drug-tracing criteria; diabetes mellitus diagnosed with laboratory data and drug- 
tracing criteria; high blood pressure, diagnosed with blood pressure measurement and drugtracing criteria; oncologic disease based on medical history, oncologic follow up and drug-tracing criteria; osteoporosis, based on bone density scan (DEXA) and drug-tracing criteria; chronic obstructive pulmonary disease (COPD), based on medical history, radiologic diagnosis and drugtracing criteria; liver disease, based on markers of viral hepatitis, echoic images and fibroscan, psychiatric illness, based on medical history, specialist evaluation and drug-tracing criteria; kidney disease: based on estimated glomerular filtration rate $(e G F R)<60 \mathrm{ml} / \mathrm{min}$; dyslipidemia based on cholesterol and triglycerides values and drug-tracing criteria.

Multi-morbidity was defined as the presence of two or more co-morbidities. The criterion of two or more chronic conditions has been considered a cutoff score to compare multi-morbid and non multimorbid samples in the total cohort.

All study participants provided informed consent to participate in the study, which was approved by the institutional ethics committee of the coordinating center and of each of the participating centers.

Statistical methods. Continuous data are presented as means (SD), or medians (IQR), and categorical data as frequency and proportions. We compared continuous data using t-tests or Wilcoxon signed -rank test and categorical data using chi-square statistics. We assumed a significance level at a p-value $<0.05$.

\section{Results}

A total of 997 patients have been enrolled. 727 (72.9\%) were males (mean age $48.5 \pm$ SD 11.1), $270(27.1 \%)$ females (46.2 \pm 9.7$)$; overall mean age was $47.9 \pm 10.8$; patients were mainly Caucasians (88.7\%); the main risk factor for HIV acquisition was heterosexual intercourse (45.6\%). Other demographics are shown in Table 1.

997 patients have been enrolled. One hundredseventy eight patients (17.8\%) had no co-morbidity, whereas 275 (27.6\%) had one condition and 544 (54.6\%) were multi-morbid: $25.8 \%$ had two comorbidities, $19.6 \%$ three, $6.6 \%$ four, $2.2 \%$ five, and $0.4 \%$ six. The most frequent co-morbidity was dyslipidemia (55.5\%), followed by bronco-pulmonary chronic obstruction (COPD) (30.0\%), hepatitis C virus (HCV) infection (25.6\%), hypertension (21.9\%),
Table 1. Characteristics of 997 patients enrolled in the Cluster Project.

SD: standard deviation; IOR: interquartile range

ART: antiretroviral treatment; eGFR: estimated glomerular filtration rate

\begin{tabular}{|c|c|c|}
\hline $\begin{array}{l}\text { CATEGORICAL } \\
\text { VARIABLES }\end{array}$ & $\mathbf{N}$ & $\%$ \\
\hline $\begin{array}{l}\text { Gender } \\
\text { Female }\end{array}$ & 270 & 27.1 \\
\hline Male & 727 & 72.9 \\
\hline Caucasian & 884 & 88.7 \\
\hline \multicolumn{3}{|l|}{ HIV risk factor } \\
\hline MSM & 274 & 27.5 \\
\hline IVDU & 208 & 20.9 \\
\hline Heterosexual & 455 & 45.6 \\
\hline Transfused & 6 & 0.6 \\
\hline Other & 54 & 5.4 \\
\hline \multicolumn{3}{|l|}{ CDC Stage } \\
\hline$A$ & 460 & 46.1 \\
\hline B & 263 & 26.4 \\
\hline C & 259 & 26.0 \\
\hline Missing & 15 & 1.5 \\
\hline ART naive & 42 & 4.2 \\
\hline $\begin{array}{l}\text { Cardiovascular events } \\
\text { before enrolment }\end{array}$ & 46 & 4.6 \\
\hline Multi-morbidity & 544 & 54.6 \\
\hline Continuous variables & Mean or median & SD or IQR \\
\hline Age & 47.8 & 10.8 \\
\hline Nadir CD4 (cells/mm3) & 203.0 & $80.0-328.0$ \\
\hline CD4 (cells/mm3) & 620.0 & $425.0-815.0$ \\
\hline Years since ART initiation & 9.0 & $4.0-16.0$ \\
\hline Serum creatinine (mg/dL) & 0.9 & 0.7 \\
\hline Glycemia (mg/dL) & 93.1 & 21.5 \\
\hline Total cholesterol (mg/dL) & 185.2 & 41.4 \\
\hline Triglycerides (mg/dL) & 119 & $84-178$ \\
\hline $\mathrm{eGFR}(\mathrm{mL} / \mathrm{min})$ & 98.0 & 27.0 \\
\hline
\end{tabular}

psychiatric illness (10.2\%) and renal impairment (4.3\%). Analyzing the pairs of co-morbidities, the most recurrent associations were: 1) dyslipidemia + $\operatorname{COPD}(177,17.8 \%), 2)$ dyslipidemia + hypertension $(158,15.8 \%)$; 3) COPD + HCV infection (134, 


\begin{tabular}{|c|c|c|}
\hline PAIRS & $\mathrm{N}=997$ & $\%$ \\
\hline Dyslipidemia+COPD & 177 & 17.8 \\
\hline Hypertension+Dyslipidemia & 158 & 15.8 \\
\hline $\mathrm{COPD}+\mathrm{HCV}$ & 134 & 13.4 \\
\hline Dyslipidemia+HCV & 129 & 12.9 \\
\hline Dyslipidemia+Cancer & 87 & 8.7 \\
\hline Hypertension+COPD & 67 & 6.7 \\
\hline Dyslipidemia+Psychiatric illness & 58 & 5.8 \\
\hline Hypertension+HCV & 50 & 5.0 \\
\hline Dyslipidemia+0steoporosis & 49 & 4.9 \\
\hline Diabetes+Dyslipidemia & 45 & 4.5 \\
\hline COPD+Psychiatric illness & 45 & 4.5 \\
\hline $\mathrm{HCV}+$ Psychiatric illness & 44 & 4.4 \\
\hline Hypertension+Cancer & 41 & 4.1 \\
\hline COPD+Cancer & 41 & 4.1 \\
\hline COPD+0steoporosis & 40 & 4.0 \\
\hline Diabetes+Hypertension & 35 & 3.5 \\
\hline Dyslipidemia+Renal impairment & 35 & 3.5 \\
\hline HCV+Osteoporosis & 31 & 3.1 \\
\hline $\mathrm{HCV}+$ Cancer & 30 & 3.0 \\
\hline Hypertension+Psychiatric illness & 23 & 2.3 \\
\hline Diabetes+COPD & 22 & 2.2 \\
\hline Diabetes+HCV & 21 & 2.1 \\
\hline Hypertension+Renal impairment & 20 & 2.0 \\
\hline Psychiatric illness+Cancer & 20 & 2.0 \\
\hline $\mathrm{HCV}+$ Renal impairment & 17 & 1.7 \\
\hline Hypertension+0steoporosis & 14 & 1.4 \\
\hline Cancer+0steoporosis & 14 & 1.4 \\
\hline Diabetes+Cancer & 13 & 1.3 \\
\hline COPD+Renal impairment & 12 & 1.2 \\
\hline Diabetes+Psychiatric illness & 9 & 0.9 \\
\hline Psychiatric illness+0steoporosis & 9 & 0.9 \\
\hline Cancer+Renal impairment & 9 & 0.9 \\
\hline Diabetes+Osteoporosis & 6 & 0.6 \\
\hline Psychiatric illness+Renal impairment & 6 & 0.6 \\
\hline Diabetes+Renal impairment & 4 & 0.4 \\
\hline Osteoporosis+Renal impairment & 3 & 0.3 \\
\hline \multicolumn{3}{|l|}{ TRIADS } \\
\hline Dyslipidemia + COPD + HCV & 68 & 6.8 \\
\hline Dyslipidemia+Hypertension+COPD & 47 & 4.7 \\
\hline Dyslipidemia+Hypertension+Cancer & 31 & 3.1 \\
\hline Dyslipidemia+Hypertension+HCV & 30 & 3.0 \\
\hline Dyslipidemia+COPD+psychiatric illness & 29 & 2.9 \\
\hline Dyslipidemia+Diabetes+Hypertension & 28 & 2.8 \\
\hline
\end{tabular}

Table 2. Co-morbidities of 997 patients enrolled in theCluster Project, by frequency.

13.4\%). Besides, HCV infection (a co-morbidity at risk of disease-disease interaction because of the potential changes in metabolism of a number of drugs of co-medication) appeared associated with hypertension in 50 (5.0\%), with psychiatric disease in $44(4.4 \%)$, with cancer in 30 (3.0\%), with diabetes in 21 (2.0\%) and with renal impairment in 17 (1.7\%). Renal impairment was also associated with dyslipidemia in 35 patients (3.5\%), COPD in $12(1.2 \%)$, hypertension in $20(2.0 \%)$, cancer in $9(0.9 \%)$, psychiatric disease in 6 $(0.6 \%)$, diabetes in $4(0.4 \%)$.

Recombining the co-morbidities in triads, we observed that the most recurrent were: dyslipidemia + COPD + HCV infection (68,6.8\%), dyslipidemia + hypertension + COPD $(47,4.7 \%)$ and dyslipidemia + hypertension + cancer $(31,3.1 \%)$.

The pairs and triads are summarized in Table 2, while their clustering modes are schematized in

\section{Figures $\mathbf{1}$ and $\mathbf{2}$.}

When we compared parameters of HIV infections, age, metabolic data and renal function between multi-morbid and non multi-morbid patients, after adjusting for age, we observed that all variables were significantly related with multi-morbidity status, except for CD4 cell count and eGFR; being naïve was not significantly different between groups, although the proportion of non experienced patients was lower in the multi-morbid group. Multi-morbid patients were older and showed lower nadir CD4 cell count, longer period in CART, higher triglycerides, glycemia, and total cholesterol. They were more frequently in CDC stage $B$ and $C$ than in stage A (Table 3).

\section{Discussion}

In our sample of patients attending the HIV-outpatient facilities of the participating centers, we found that most PLHW were multi-morbid and about 30\% had three or more concurrent conditions.

Comparable data with respect to the numbers of patients with two or more concurrent medical conditions and their clusterization are scarce because of heterogeneous definitions of multi-morbidity $(3,4)$. In particular, few studies have been performed among PLWH. Comparing co-morbidity among a large cohort of veterans with and without HIV infection, Goulet et co-workers (5) found that co-morbidity is the rule, and multi-morbidity is common among veterans with HIV infection. Patterns of comorbidity differ substantially by HIV status, age, and HIV severity. In multivariable 


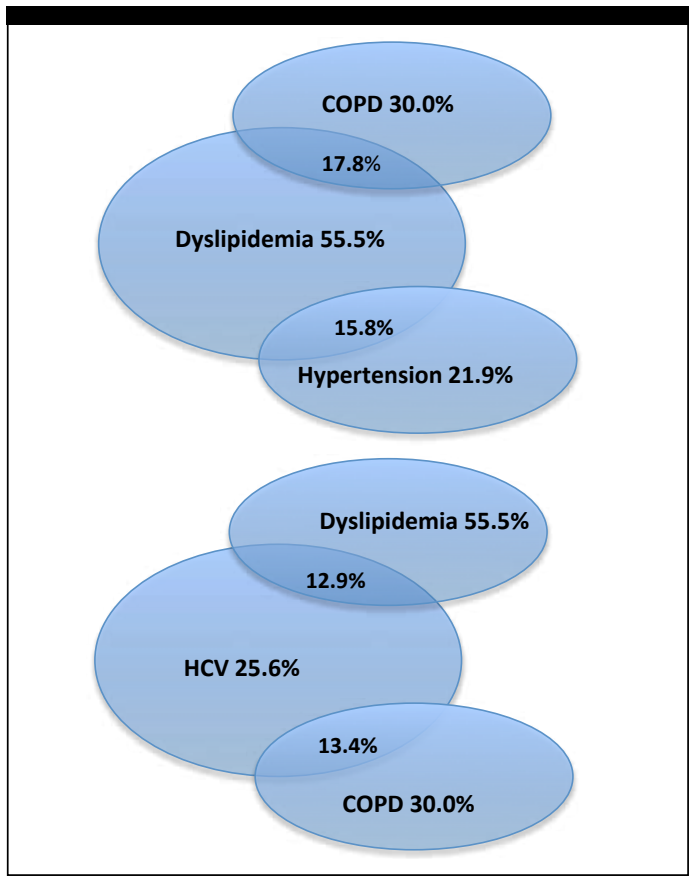

Figure 1. Clustering mode of the most frequent pairs of observed co-morbidities.

analyses, older HIV-infected veterans were more likely to have substance use disorder and multimorbidity. Renal, vascular, and pulmonary diseases were associated with CD4 cell count $<200$ cells/mm3; hypertension was associated with CD4 cell count $>200$ cells/mm3. Kim and co-workers (6) performed a study on a population of PLWH examining the relationship with obesity and multi-morbidity. They found that prevalence increased with progressive BMI categories. Three multi-morbidity clusters were identified: "Metabolic" including hypertension, gout, diabetes mellitus, and CKD; "Behavioral" including mood disorders, dyslipidemia, COPD, chronic ulcer disease, osteoarthritis, obstructive sleep apnea, and cardiac disorders; "Substance Use" including alcohol abuse, substance abuse, tobacco abuse, and hepatitis C. Obesity was associated with a higher likelihood of multi-morbidity. Interestingly, Markun and coworkers (7) performed a study with the aim to identify therapeutic conflicts in emergency department HIVnegative patients with multi-morbidity. In his paper major therapeutic conflict was defined as a situation where clinical practice guidelines recommend a treatment of one medical condition that is absolutely contraindicated because of a coexisting condition (for example, a situation where anticoagulation is recommended because of a pulmonary embolism, but at the same time contraindicated because of a coexisting gastrointestinal bleeding). Minor therapeutic conflict was defined as a where clinical practice guidelines recommend a treatment of one medical condition that is relatively contraindicated because of

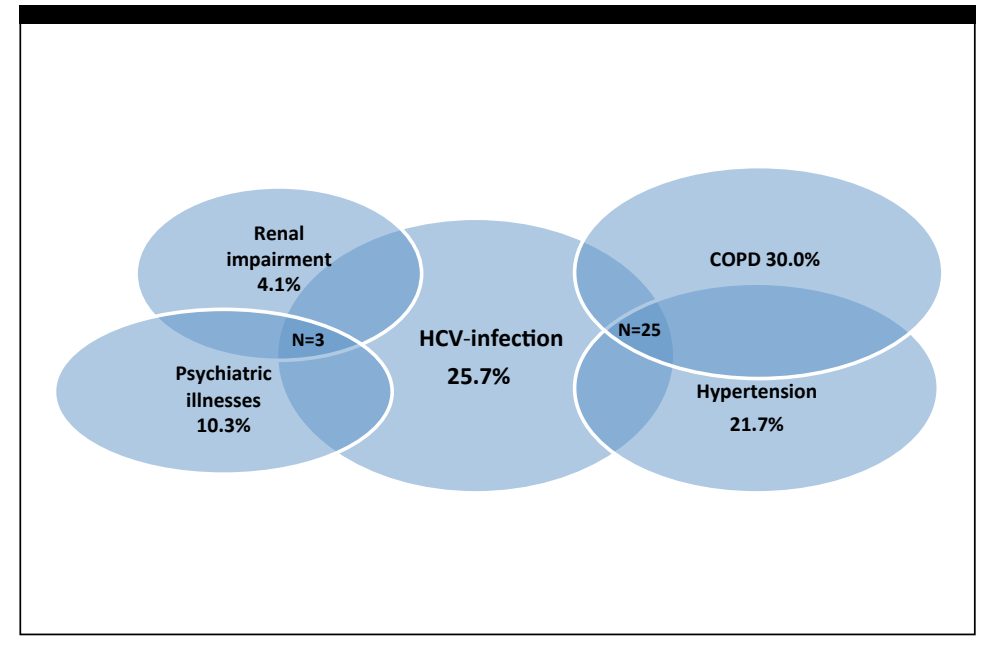

Figure 2. Clustering mode of triads of particular interest.

Table 3. Comparison of parameters of HIV infections, age, metabolic data and renal function between multi-morbid and non multi-morbid patients.

* all statistical significance (but age itself) are age-adjusted

\begin{tabular}{|c|c|c|c|}
\hline & $\begin{array}{c}\text { MULTI- } \\
\text { MORBIDITY }\end{array}$ & $\begin{array}{c}\text { No multi } \\
\text {-morbidity }\end{array}$ & $\mathbf{p}^{*}$ \\
\hline Age & $51.7 \pm 9.2$ & $43.4 \pm 10.9$ & $<0.0001$ \\
\hline Gender (M) & $413(75.9 \%)$ & $314(69.3 \%)$ & 0.22 \\
\hline ART naïve & $10(1.8 \%)$ & $31(6.8 \%)$ & 0.14 \\
\hline \multicolumn{4}{|l|}{ CDC stage } \\
\hline A & $200(37.2 \%)$ & $259(57.7 \%)$ & ref. \\
\hline$B$ & $159(29.6 \%)$ & $109(24.3 \%)$ & 0.63 \\
\hline C & $178(33.2 \%)$ & $81(18.0 \%)$ & 0.0006 \\
\hline Nadir CD4 (cells/mm3) & $165(70-290)$ & $240(100-360)$ & 0.013 \\
\hline CD4 (cells/mm3) & $627(428-840)$ & $614(422-776)$ & 0.11 \\
\hline $\begin{array}{l}\text { Years since } \\
\text { ART initiation }\end{array}$ & $12(6-17)$ & $6(2-12)$ & $<0.0001$ \\
\hline Glycemia (mg/dL) & $96.5 \pm 26.3$ & $88.9 \pm 12.4$ & 0.002 \\
\hline Total cholesterol (mg/dL) & $190.3 \pm 42.9$ & $178.7 \pm 37.9$ & 0.002 \\
\hline Triglycerides (mg/dL) & $145(96-201)$ & $99(70-137)$ & $<0.0001$ \\
\hline eGFR (mL/min) & $94.7 \pm 28.3$ & $101.6 \pm 24.8$ & 0.96 \\
\hline
\end{tabular}

a co-existing condition (for example, a situation where acetylsalicylic acid is recommended because of a vascular disease, but at the same time contraindicated because of a co-existing reflux esophagitis), but where the treatment is possible without adverse effects if certain precautions are taken. The Authors identified a number of mayor and minor therapeutic conflicts due to disease-disease interactions. Among mayor conflicts they found: cancer requiring chemotherapy or immunosuppression in patients with renal failure, 


\section{original article}

or with cirrhosis at risk of gastrointestinal bleeding; and CVD requiring acetylsalicylic acid or oral anticoagulants in cirrhotic patients with disturbances of coagulation. Among minor conflicts they found: CVD requiring diuretic treatment in patients with renal failure; CVD requiring antihypertensive agents in patients with renal failure or with cirrhosis at risk of gastrointestinal bleeding; CVD in patients requiring beta blockers in patients with asthma. Most of these disease-disease interactions are not infrequent in PLWH, but we are not aware of similar studies among these patients.

Our data evidence that, in spite of mean age lower than 50 , co-morbidity is the rule among PLWH (82.2\%), and that multi-morbid patients are the majority (54.6\%). Moreover, about $30 \%$ of them had three or more chronic non-HIV related conditions, this confirming recent data provided by other studies in this field (10). Dyslipidemia was by far the most frequent comorbidity but HCV-related hepatitis with detectable levels of HCV-RNA (both treatment naives and therapy failed) still remains very frequent in spite of the big efforts under way to cure this co-infection. In the light of the study of Markun (7), the chronic condition that appear most at risk for disease-disease interactions are liver disease, renal impairment and cardiovascular diseases. In our experience, both in pairs and in triads they appear frequently associated with other comorbidities, and among them. All the conditions potentially at risk for mayor and minor disease-disease interactions suggested by Markun (respectively cancer + renal impairment, cancer + liver disease, CVD + liver disease, and CVD + renal impairment, CVD + liver disease, CVD + COPD) were present among our patients, although in different percentages. Not unexpectedly, multi-morbid patients presented higher mean age, worst parameters of HIV infections, more compromised metabolic data and renal function with respect to non multi-morbids.

Few studies address the problem of clusterization of chronic non communicable conditions among PLWH and, although other cohorts examine a higher number of patients respect to our experience, data were extracted from diagnosis codes, like ICD-9-CM (5), or from electronic health records (6). In our study data were provided by the infectious disease specialists who followed their patients, this providing more reliable and updated data. Moreover, at the best of our knowledge, this is the first effort to identify potential disease-disease interactions among PLWH.

Possible limitations of our study are the absence of a control group of HIV-negative subject. Moreover, the investigation is aimed at the potential disease-disease interactions: a liver disease do not necessarily means a decompensated cirrhosis, such as a risk factor for CVD do not necessarily means an overt cardiac disease. Further data are warranted to identify the patients actually exposed to the risk of disease-disease interactions. However, considering the aging of PLWH, their persistent exposure to chronic inflammation and the possible effects of HAART, it is mandatory to face since now the phenomenon of clusterization, and to identify all the patients at risk of disease-disease interactions. The identification of common patterns of comorbidities in PLWH will help to identify and address the combined risks of increasingly prevalent multiple morbidities and disease-disease interactions.

\section{BIBLIOGRAFIA}

1. Krentz HB, Kliewer G, Gill MJ. Changing mortality rates and causes of death for HIV-infected individuals living in Southern Alberta, Canada, from 1984 to 2003. HIV Med 2005; 6: 99-106.

2. Grinzstejn B, Luz PM, Pacheco AG et al. Changing mortality profile among HIV-infected patients in Rio de Janeiro, Brazil: shifting from AIDS to non-AIDS related conditions in the HAART era. PLoS One 2013; 8: e59768.

3. Fortin $\mathrm{M}$, Stewart M, Poitras ME, Almirall J, Maddocks H. A systematic review of prevalence studies on multimorbidity: toward a more uniform methodology. Ann Fam Med 2012; 10: 142-51.

4. 2. Valderas J, Starfield B, Sibbald B, Salisbury C, Roland M. Defining comorbidity: implications for understanding health and health services. Ann Fam Med. 2009; 7: 357-63.

5. Goulet IL, Fultz SL, 3, Rimland D, et al. Do Patterns of Comorbidity Vary by HIV Status, Age, and HIV Severity? Clin Infect Dis. 2007; 45: $1593-601$.

6. Kim DJ, Westfall AO, Chamot E, Willig AL, Mugavero MJ, et al. Multimorbidity Patterns in HIV-Infected Patients: The Role of Obesity in Chronic Disease Clustering. J Acquir Immune Defic Syndr . 2012; 61: 600-5.

7. Markun S, Holzer BM, Rodak R, et al. Therapeutic Conflicts in Emergency Department Patients with Multimorbidity: A Cross Sectional Study. PLoS One 2014; 9: e110309.

8. Maciel RA, Klück HM, Durand M, Sprinz E. Comorbidity is more common and occurs earlier in persons living with HIV than in HIVuninfected matched controls, aged 50 years and older: A cross-sectional study. Int J Infect Dis. 2018; 70: 30-5. 